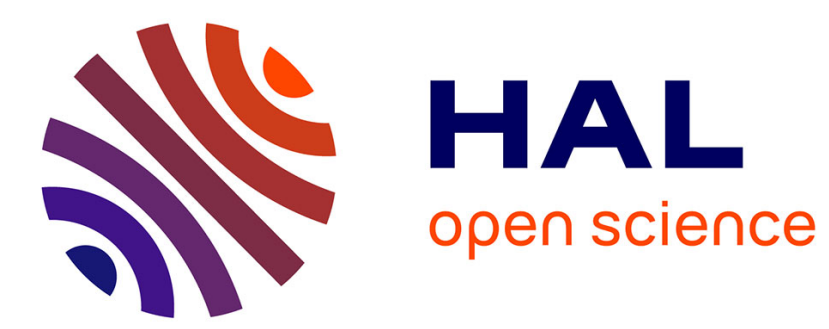

\title{
Introduction au dossier sur les retraites: un débat pour progresser
}

Jean-Paul Fitoussi

\section{To cite this version:}

Jean-Paul Fitoussi. Introduction au dossier sur les retraites: un débat pour progresser. Revue de l'OFCE, 1999, 68, pp.9 - 14. 10.3406/ofce.1999.1527 . hal-03048981

\section{HAL Id: hal-03048981 \\ https://hal-sciencespo.archives-ouvertes.fr/hal-03048981}

Submitted on 9 Dec 2020

HAL is a multi-disciplinary open access archive for the deposit and dissemination of scientific research documents, whether they are published or not. The documents may come from teaching and research institutions in France or abroad, or from public or private research centers.
L'archive ouverte pluridisciplinaire HAL, est destinée au dépôt et à la diffusion de documents scientifiques de niveau recherche, publiés ou non, émanant des établissements d'enseignement et de recherche français ou étrangers, des laboratoires publics ou privés. 


\title{
Introduction au dossier sur les retraites : un débat pour progresser
}

\author{
Jean-Paul Fitoussi \\ Président de l'OFCE
}

L'avenir des systèmes de retraite est un sujet essentiel dans ses trois dimensions, humaine, sociale et économique. Cet avenir, à tort ou à raison, apparaît sombre pour l'instant, sous l'effet conjugué de deux nouvelles, l'une bonne, l'autre qui l'est moins : l'espérance de vic est élevée et continue de s'accroître: les évolutions démographiques sont par ailleurs telles que le ratio du nombre de retraités au nombre d'actifs va augmenter de $38 \%$ environ aujourd'hui à $50 \%$ en 2020 , pour atteindre $70 \%$ en 2040, en supposant inchangé l'âge de la retraite. Ces données sont de nature à inquiéter les populations car elles ne peuvent que conduire à l'anticipation d'une baisse du niveau de vie pendant la retraite. L'immobilisme et l'absence d'engagement clair de l'Etat auraient, dans ces circonstances, des effets pervers. Mais il n'est nul besoin d'inquiéter (en publiant des chiffres alarmistes), pour agir. Cela ne peut qu'aggraver la perte de confiance des français dans le système de solidarité mis en place en 1945. L'incertitude croît en effet parmi les générations du baby-boom qui subissent l'assaut de compagnies d'assurances et de banques cherchant, par tous les moyens, (y compris par des campagnes publicitaires à la limite du mensonge et de l'intimidation) à placer leurs produits d'épargne. Les grandes entreprises ont aussi trouvé un moyen de s'attacher leurs salariés, particulièrement les cadres, à moindre coût, en développant des produits financiers " maison » offrant des garanties de retraites surcomplémentaires. L'idée de la faillite inéluctable des régimes de répartition s'installe peu à peu. Alors que la logique même de ces régimes - les actifs payent directement les retraites - offre la protection la plus sérieuse aux futurs retraités en gageant les prestations sur les salaires courants. Il résulte de cette évolution une inquiétude et une défiance propices à la recherche de solutions individuelles. Ceux qui en ont les moyens épargnent à long terme, non pas en vue d'accroître leur bien-être futur par la consommation ou l'investissement, mais pour se prémunir contre la chute annoncée des retraites.

Une telle situation ne créé pas un climat favorable à la croissance, qui implique des anticipations positives, une attitude tournée vers le progrès plutôt que motivée par la crainte de l'avenir. Mais surtout, elle porte en germe le développement d'inégalités sociales nouvelles : alors que le 
système de retraites avait permis de stabiliser les inégalités entre personnes âgées au niveau des inégalités entre actifs, le recours à l'épargne individuelle jouera nécessairement comme un amplificateur d'inégalités au moment de la retraite. Les plus favorisés dans la vie active verront leur position relative s'élever encore au moment de la retraite, puisqu'ils auront eu la possibilité de se constituer une épargne à la différence des plus pauvres.

Une rapide intervention du politique permettra de clarifier les règles du jeu du système de retraite à long terme et de "restaurer la confiance $"$.

1. La mise en place d'un étage de capitalisation universel et obligatoire au sein de nos systèmes de retraite peut constituer un élément de la reconquête des esprits en faveur de la solidarité collective. Cependant, si la capitalisation est une solution individuelle, elle ne peut pas, à l'échelle de la société, réduire la contribution des actifs au financement des retraites et se substituer aux transferts intergénérationnels directs (entre générations actives et retraitées vivant au même moment). Dans toutes les hypothèses, la production résulte de l'activité et doit être partagée entre les personnes au travail et les retraités. Ce partage répond à des considérations indépendantes du régime de retraite choisi, qui peuvent être de solidarité, de cohésion sociale, de respect pour les plus âgés etc. En 2050, par exemple, le nombre des retraités sera approximativement égal à celui des actifs, selon les projections les plus alarmistes. Quel que soit le système de retraite, il faudra bien consacrer une part importante de la production courante aux plus âgés. Cette part sera déterminée davantage par des motifs culturels et anthropologiques (la structure familiale, notamment) que par des considérations techniques ou économiques. Que le système soit de répartition ou de capitalisation, les actifs de demain pourront toujours en effet s'arranger pour réduire la part qui revient aux retraités, en refusant toute hausse de cotisation dans le premier cas, ou en créant une situation inflationniste dans le second. Dans les deux cas, ils prennent aussi le risque d'être traités de même façon par les générations à venir. La solidarité entre générations n'est qu'en apparence plus forte dans un système par répartition que dans un système par capitalisation.

La raison en est simple : quand un individu épargne en vue de la retraite, il acquiert en fait des droits sur le produit du travail des générations futures. C'est ce travail, et lui seul, qui permettra de rémunérer son épargne lorsqu'il aura cessé toute activité de production. A chaque instant, sauf à renier leurs parents et à accepter le développement de la pauvreté parmi les retraités, les générations actives ne peuvent éviter de travailler en partie pour assurer la consommation des générations inactives (retraités, mais aussi enfants, étudiants, handicapés,...). Mais il ne s'agit pas, comme on pourrait trop facilement le penser, d'un jeu à somme nulle. Il est possible d'augmenter le volume de la production à partager, 
en investissant. La seule manière de transférer des ressources vers l'avenir, en effet, consiste à accumuler du capital productif sous toutes ses formes - capital de production direct (machines), connaissances, formation, équipements publics - ce qui permettra aux générations futures de produire, pour elles-mêmes et pour leurs parents retraités, des biens en quantité telle que le "partage ", inévitable, soit celui de l'abondance et non celui de la pénurie. Le moyen du partage est d'une importance secondaire : que les actifs subissent un prélèvement sur leurs salaires ou qu'ils doivent rémunérer ou racheter le capital détenu par leur parents ne modifie pas la part qu'ils pourront, in fine, affecter à leur propre consommation.

2. S'il n'est pas possible d'échapper aux transferts intergénérationnels instantanés (transferts entre générations vivant au même moment). le montant de ces transferts dépend directement du rapport entre inactifs et actifs et s'élève nécessairement lorsque ce rapport augmente. Pour les générations nées depuis la guerre, les dés sont jetés : la charge relative des retraites sur les actifs des années 2000-2050 sera plus importante que celle qui pesa sur leurs parents.

Mais le montant de cette charge peut faire l'objet d'un débat : la durée de la vie active peut être allongée, le niveau moyen des retraites révisé (dans la ligne de la réforme Veil-Balladur de 1993 pour le régime général). Pour les salariés du bas de l'échelle, la réduction du montant des retraites serait inéquitable. Les réformes déjà appliquées (allongement de la durée de cotisations, réduction du montant des pensions, baisse des rendements des régimes complémentaires) devraient réduire de 5 à 10 points le taux de remplacement du revenu d'activité par les retraites (montant moyen de la retraite / dernier salaire). La marge de manœuvre sur le niveau des retraites est donc très limitée.

Reste l'âge du départ à la retraite : un an de recul en moyenne représente une économie de $5 \%$ sur les prestations. Si, par exemple, l'âge de la retraite était porté à 65 ans, le ratio du nombre des retraités à celui des actifs serait en 2020 inférieur à ce qu'il est aujourd'hui où l'âge de la retraite est à 60 ans, ce qui permettrait une baisse des cotisations. En 2040, toutefois, l'augmentation des cotisations redeviendrait inévitable, mais dans une proportion bien moindre que dans l'hypothèse où une telle réforme n'était pas mise en œuvre. Dans un contexte où l'espérance de vie est élevée et où les gens vivent longtemps en bonne santé, qu'estce qui s'oppose au recul de l'âge de la retraite? La réponse est évidemment l'état du marché du travail, la situation de chômage de masse persistant dans laquelle se trouve la France depuis plus d'une décennie. On sait qu'une telle situation touche de façon disproportionnée les âges extrêmes : les jeunes et les "vieux ». S'il n'était pas porté remède au chômage, le recul de l'âge de la retraite ne servirait à rien. Cela reviendrait à demander aux gens de travailler plus longtemps alors qu'ils manquent déjà de travail. Par contre l'emploi des catégories les plus âgées de 
la population active est grandement facilité lorsqu'il n'existe pas de chômage parmi les jeunes. On le voit, résoudre la question du chômage participerait de façon décisive à la solution du problème des retraites.

Pour restaurer la confiance dans le système de retraites, il convient donc de fixer rapidement les paramètres qui seront appliqués au cours de vingt prochaines années : taux de retraites et âge moyen normal de sortie de la vie active. Ce n'est qu'une fois ces paramètres choisis qu'il sera possible d'évaluer la hausse nécessaire des cotisations, en particulier à un régime nouveau de capitalisation obligatoire. Mais il convient aussi de dépassionner le débat. Si rien n'était fait, nous disent les augures les plus pessimistes, il faudrait que les taux de cotisations doublent d'ici 2040 pour atteindre $40 \%$. C'est certes considérable, et l'on peut imaginer les effets d'incitation négatifs qui résulteraient d'une augmentation aussi forte des prélèvements obligatoires. Mais il faut aussi tenir compte de l'évolution des niveaux de vie : en 2040, le PIB français sera environ 2,5 fois plus élevé que ce qu'il est aujourd'hui. Malgré l'augmentation des cotisations, les français, y compris les retraités, seront à cette date beaucoup plus riches. Leur revenu net aura simplement moins augmenté que leur revenu brut. L'enjeu est donc sérieux, mais il est nécessaire de le ramener à sa juste proportion pour que l'on puisse débattre de façon sereine des solutions les meilleures.

3. Les propositions de constitution de fonds de pensions peuvent être séduisantes si elles procèdent d'un idéal de justice et d'équité. En ce cas, le régime devrait être universel, obligatoire et plus favorable aux basses rémunérations qu'aux rémunérations élevées. Ainsi, non seulement, les fonds de pension ne contribueraient-ils pas à la croissance des inégalités, comme on le pense d'ordinaire, mais au contraire, ils permettraient de les réduire.

L'observation empirique des rendements des placements financiers rend, d'autre part, la capitalisation alléchante pour financer la hausse future des dépenses de retraite. Mais peut-on penser qu'à long terme, et seul le long terme importe en la matière, ces rendements demeureront aussi élevés? La plupart des pays occidentaux sont logés à la même enseigne que la France, encore que dans notre pays le vieillissement de la population soit moindre qu'ailleurs. Si donc tous les pays de l'OCDE. faisaient de concert un effort d'épargne supplémentaire pour régler la question des retraites, pourrait-on être sûr que le rendement de l'épargne n'en serait point affecté? La réponse donnée par l'analyse économique est qu'il s'en suivrait une baisse des taux d'intérêt et du rendement du capital. Mais tout dépend de l'ampleur de l'augmentation de l'épargne et de l'importance des nouvelles opportunités d'investissement qui s'offriront. Au total, on le voit, il n'est pas de l'ordre de la certitude que les régimes de retraite par capitalisation conserveront dans l'avenir le même avantage qu'ils ont aujourd'hui relativement aux systèmes par répartition. Cet avantage provient de ce que, depuis le début 
des années quatre-vingt le taux d'intérêt réel est nettement plus élevé que le taux de croissance.

Par contre, l'argument selon lequel les fonds de pension favoriseraient le développement des entreprises m'a toujours semblé insuffisamment fondé. Cela provient probablement d'une incompréhension de ma part. Ce qui favorise le développement des entreprises, c'est l'abondance de l'épargne. Or le taux d'épargne des ménages est en France plus élevé qu'aux Etats-Unis. Que fait le système financier de cette abondance d'épargne? Pourquoi ne l'a-t-il pas investi dans les entreprises, si la rentabilité de ces dernières est aussi élevée qu'on le dit? Il y a là quelque chose qui m'apparaît bien mystérieux, car aussi bien cette épargne ne s'est pas évanouie. Comment être sûr que les fonds de pension adopteraient un comportement différent du système financier actuel, en plaçant de façon privilégiée leurs ressources dans les PME?

La constitution d'un étage supplémentaire de capitalisation est une opération délicate si elle n'est pas purement homéopathique, car le contexte actuel n'est pas celui d'une insuffisance d'épargne. Les années quatre-vingt-dix ont plutôt été caractérisées par des taux d'épargne élevés, un excédent des échanges extérieurs et une anémie de la demande de consommation et d'investissement. Or, pour que la capitalisation puisse contribuer significativement au financement des retraites de 20202030 , il faudrait instituer dès maintenant une cotisation de l'ordre de $10 \%$ sur les salaires dans l'hypothèse d'un maintien des règles courantes de calcul des retraites. Si l'âge de la retraite était reculé à 65 ans, la cotisation pourrait être limitée à $5 \%$ environ.

On peut penser que pour les cadres, l'institution d'un système de capitalisation obligatoire se traduira par une réduction de l'effort d'épargne individuelle. Mais pour la grande majorité des salariés, ce prélèvement supplémentaire provoquera nécessairement une baisse de la consommation. Pour stabiliser le niveau de la demande globale, il faudrait donc réduire en contrepartie un autre prélèvement et accepter un déficit public supplémentaire. Son financement serait aisé en raison de la hausse « obligatoire » de l'épargne et serait donc sans effet sur le taux d'intérêt. Il serait sans doute préférable de baisser les taux d'intérêt, plutôt que d'augmenter le déficit budgétaire, afin de compenser l'effet récessif sur la demande globale de la cotisation au régime de capitalisation. Mais aucune de ces mesures ne semble possible aujourd hui.

La hausse des prélèvements qu'implique un système de capitalisation est d'une autre nature que celle qui doit résulter du contre-choc démographique de 2005-2010: dans le cas de la capitalisation il faut anticiper la hausse et. pendant la phase de montée en charge du nouveau régime, il n'existe aucune contrepartie du côté des prestations. Par contre, si l'on s'en tient au seuls régimes par répartition, l'augmentation des cotisations interviendra seulement au moment du décollage des prestations. sans effets sur la demande globale. 
En matière de retraite on ne peut que s'accorder avec l'idée qu'il est indispensable de prévoir. Et la tentation est parfois grande d'assimiler la prévoyance à l'épargne. Mais prenons garde de ne pas épargner l'argent de l'essence si nous souhaitons arriver à l'étape suivante du voyage.

Que conclure? Les fonds de pension sont dans l'air du temps. Leur développement semble même inéluctable car ils sont à l'intersection d'un problème et d'une évolution. Le problème, c'est bien sûr celui de l'avenir des retraites; l'évolution, c'est celle de l'économie de marché dans le contexte de la mondialisation. L'effondrement du mur de Berlin a institué le modèle américain en référence universelle, et en ce pays les fonds de pension jouent un rôle dominant. Mais sont-ce des raisons suffisantes pour s'embarquer dans la même aventure? J'ai émis certaines réserves, exprimé certains doutes, souligné certains risques tant il me semble que la question mérite encore d'être débattue.

Je suis sans réserve pour qu'un débat clair, transparent, sérieux s'instaure en permanence sur les questions de société les plus importantes. Seule la pratique du débat peut permettre de progresser dans la solution des problèmes de notre temps. Elle permet de transcender les a priori inévitables lorsque l'on s'empare d'un sujet, de corriger ses propres erreurs, et même parfois d'aboutir à un véritable consensus, c'est-à-dire qui ne soit pas de renoncement, mais au contraire de progrès. Car il est par ailleurs des consensus "mous " qu'il vaut mieux éviter. Les deux articles suivants, I'un portant sur le problème des retraites en France et l'autre en Allemagne, représentent une contribution de I'OFCE à ce grand débat. Ils sont précédés, pour les lecteurs intéressés par l'analyse comparative. par une note permettant de faciliter la comparaison des régimes de retraite dans les deux pays. 